\title{
Análise descritiva da mortalidade por câncer de pulmão no município de São Paulo de 2005 a 2014
}

\author{
Descriptive analysis of lung cancer mortality in the municipality of São Paulo from \\ 2005 to 2014
}

\author{
Igor Bastos Polonio ${ }^{1}$, Maria Josefa Penon Rujula ${ }^{2}$, Gabriela Alves Loyo ${ }^{3}$, Talita Carla Stratti Moreira ${ }^{3}$
}

\section{Resumo}

Objetivos: Analisar o câncer de pulmão e brônquios de acordo com sexo, idade e localização no Município de São Paulo para facilitar desenvolvimento e implementação de políticas públicas de prevenção e combate à doença. Métodos: Análise de dados extraídos do Sistema de Informação de Mortalidade - SIM do DataSus, referentes a pacientes a partir de 0 anos de idade que faleceram em razão da doença no Município de São Paulo, no período de 2005 a 2014. Resultados: A mortalidade foi maior em pacientes do sexo masculino em todas as faixas etárias estudadas, mas especialmente maior entre aqueles acima dos 50 anos de idade. Quanto a localidade, nota-se um coeficiente de mortalidade maior nos distritos administrativos mais ricos de São Paulo. Conforme aumenta a renda da população, maior é o número de fumantes, pois são regiões que possuem mais recursos para financiar o hábito por tabaco. Conclusão: Os dados demonstraram que sexo, idade e localização residencial, associados a tabagismo, exposição ocupacional e poluição atmosférica; influenciaram a taxa de mortalidade no período estudado. Assim, evidencia-se a necessidade de politicas de controle e/ou preservação contra os fatores predisponentes, como forma de diminuir a incidência da doença.

Descritores: Neoplasias pulmonares, Pulmão, Brasil, Distribuição por sexo e idade, Distribuição espacial da população, Mortalidade

1. Professor Assistente da Faculdade de Ciências Médicas da Santa Casa de São Paulo - Departamento de Clínica Médica. Professor de Medicina da Faculdade de Medicina da Universidade Anhembi Morumbi

2. Professora Assistente da Faculdade de Ciências Médicas da Santa Casa de São Paulo - Departamento de Saúde Coletiva

3. Estudante de Medicina da Universidade Anhembi Morumbi

Trabalho realizado: Escola de Ciências da Saúde. Universidade Anhembi Morumbi

Endereço para correspondência: Igor Bastos Polonio. Rua Monte Alegre, 47, Perdizes -05014-000 - São Paulo-SP - Brasil. Tel. 5511 3862-5081.E-mail:igbpolonio@yahoo.com.br

\begin{abstract}
Objective: To analyze lung and bronchial cancer according to sex, age and location in the Municipality of São Paulo to facilitate the development and implementation of public policies to prevent and combat disease. Method: Analysis of data extracted from the "Sistema de Informação de Mortalidade - SIM" (Mortality Information System), from DataSus, was studied, as long as were related to patients, starting from 0 years old, who died in the city of Sao Paulo, between 2005 and 2014. Results: Mortality was greater in male patients in all age ranges studied but especially high among those above the age of 50. Regarding location of residence, one can observe a greater death coefficient in the districts with higher acquisitive power in Sao Paulo. The higher the acquisitive power, the greater was the number of smokers, having more resources to finance the habit. Conclusion: The data showed that gender, age and residence location associated with tabagism, occupational exposure and air pollution; influenced the mortality rate in the studied period. Therefore, the need of Control and/ or Prevention Policies is evident, as means to reduce the incidence of the disease.
\end{abstract}

Keywords: Lung neoplasms, Lung, Brazil, Age and sex distribuition, Residence characteristics, Mortalitity

\section{Introdução}

O câncer é um problema de saúde pública mundial. Compreende um grupo de doenças caracterizadas pela rápida proliferação de células anormais.

O câncer de pulmão, especificamente, ocorre a partir da proliferação e disseminação descontrolada de células anômalas nos tecidos do pulmão, um dos tipos de câncer que mais matam no mundo; responsável por mais de 1,59 milhões de mortes, seguido pelo câncer de fígado (745 mil mortes) e estômago

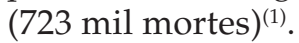

Esse tipo de tumor é responsável por 1 em cada 5 mortes por câncer no mundo (1.550.000 mortes, 19,4\% do total) ${ }^{(1)}$. 
Em 2012, um levantamento realizado pela Organização Mundial de Saúde - OMS mostrou uma incidência maior no sexo masculino mundialmente falando, sendo 1,82 milhão o total $(12,9 \%$ do total de casos estudados), destes 1,24 milhão homens e 583 mil mulheres ${ }^{(1)}$.

No Brasil, o tumor maligno de pulmão é o segundo mais frequente na população masculina nas regiões Sul $(33,62 / 100$ mil) e Centro-Oeste (14,03/100 mil), segundo os dados da Estimativa de 2014 do Instituto Nacional do Câncer - INCA ${ }^{(2)}$. No Sudeste $(18,51 / 100$ mil), Nordeste $(9,01 / 100$ mil) e Norte $(7,69 / 100$ mil), correspondem é ao terceiro mais frequente ${ }^{(2)}$.

Em relação ao sexo feminino, no Sul (21,35/100 mil) e Sudeste (11,48/100 mil), é o terceiro mais comum. Nas demais regiões, Centro-Oeste (8,49/100 mil) e Nordeste $(6,40 / 100 \mathrm{mil})$ e Norte $(5,11 / 100 \mathrm{mil})$, correspondem respectivamente, ao quarto e quinto mais prevalentes ${ }^{(2)}$.

A neoplasia pulmonar está associada a diversos fatores de risco, como: asbestos, arsênico, poluição atmosférica, radônio, histórico familiar de neoplasia pulmonar, doença pulmonar prévia, exposição à radiação, fumante passivo, Lúpus Eritematoso Sistêmico, sistema imunológico deprimido e tabagismo, sendo o último o de maior importância para o desenvolvimento da doença ${ }^{(3)}$.

O tabagismo é reconhecido como uma doença epidêmica pela OMS, matando cerca de 200 mil pessoas por ano no Brasil, equivalente a 23 pessoas por hora, segundo o INCA ${ }^{(4)}$. É um fator de risco para mais de 50 doenças, sobretudo para diversos cânceres, especialmente o de pulmão. Juntamente com o câncer de laringe, esses dois cânceres associados ao tabagismo correspondem a $12,3 \%$ dos tipos de câncer no país ${ }^{(4)}$.

De acordo com a OMS, o fumo de cigarros mata 5,4 milhões de pessoas anualmente. Estima que serão 8 milhões de mortes, até 2030 , sendo $80 \%$ em países em desenvolvimento ${ }^{(5)}$.

Estudos da American Cancer Society afirmam que cessar o tabagismo em qualquer idade pode reduzir o risco de morte e que parar de fumar por volta dos 40 anos reduz a mortalidade em cerca de $90 \%{ }^{(5)}$.

Nos Estados Unidos, de 2005 a 2010 houve 951.454 óbitos por neoplasia de brônquios e pulmonar. Enquanto que na África do Sul e Reino Unido, no mesmo período o número de óbitos foi de 27.801 e 207.463, respectivamente. Na Austrália morreram cerca de 46.918 pessoas, no período de 2006 a $2011^{(6)}$.

A mortalidade do câncer de pulmão tem um impacto importante no Brasil. No país, em 2005, houve 18.022 óbitos por câncer de pulmão e brônquios, enquanto que no ano de 2011, o número de óbitos foi de 22.346. No período de 2005 a 2011 morreram por esse mesmo câncer cerca de 142.108 pessoas, sendo 90.344 homens e 51.764 mulheres $^{(6)}$.
Não há estudos sobre a mortalidade do câncer de pulmão no município de São Paulo, por isso o presente trabalho tem o intuito de conhecer a mortalidade deste câncer na cidade mais populosa do Brasil. Será estudada a mortalidade da neoplasia pulmonar relacionada ao gênero, faixa etária e local de residência dos pacientes no município de São Paulo.

\section{Métodos}

O presente trabalho é um estudo descritivo, realizado por meio de análise de dados referentes a pacientes que residiam do município de São Paulo e que vieram a óbito em função de Neoplasia pulmonar e de brônquios.

Quanto as análises, as comparações do coeficiente de mortalidade por sexo, faixa etária e distritos administrativos foram realizadas através de gráficos de barra de erro com média e erro padrão. A comparação da mortalidade por gênero nas diferentes classes de idade foi avaliada pelo teste de Mann-Whitney após teste de heterocedasticidade (Teste de Levene). A associação entre a faixa etária e o coeficiente de mortalidade foi avaliada por um modelo de regressão exponencial.

Os dados analisados foram obtidos do Sistema de Informação de Mortalidade (SIM), do DATASUS, localizados no site da Secretaria Municipal da Saúde da prefeitura de São Paulo, dados estes de domínio público.

A partir desses dados foi elaborada fórmula para cálculo de coeficiente de mortalidade, conforme abaixo:

Número de Óbitos $\div$ Número da População × 100.000

Restringiu-se a pesquisa ao período de janeiro de 2005 a dezembro de 2014, dividindo entre pacientes do sexo masculino e feminino, a partir de 0 anos em intervalos de 5 anos até 75 anos e mais e conforme o distrito administrativo.

Tratando-se de dados secundários retirados do Sistema de Informação sobre Mortalidade - SIM, sem citar ou identificar pacientes, dispensou-se o uso do Termo de Conhecimento Livre e Esclarecido - TCLE, seguindo as normas da Comissão de Ética Pública CEP.

\section{Resultados}

A mortalidade nos homens obteve uma média de 18,72 (erro padrão $\pm 0,24$ ) e nas mulheres 11,46 (erro padrão $\pm 0,38$ ) (Figura 1).

A correlação entre a faixa etária e a mortalidade foi muito alta e foi ajustado um modelo matemático de função exponencial $(y=0,0077 \mathrm{e} 0,1377 \mathrm{x})$ que com- 


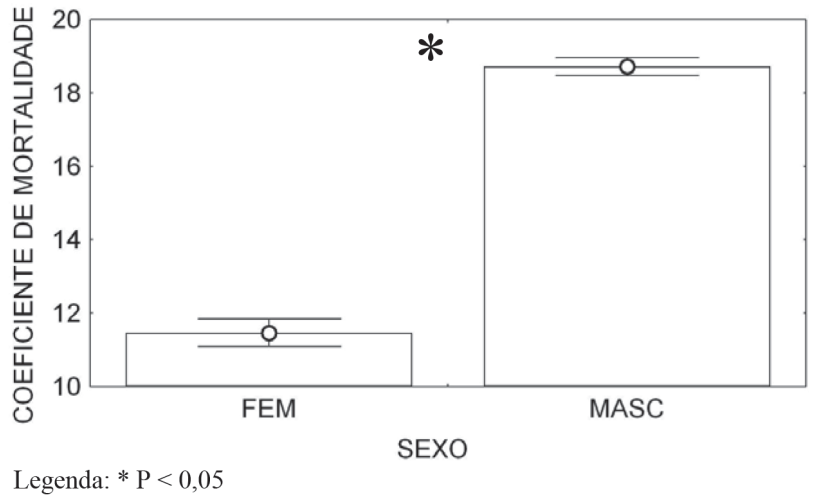

Figura 1 - Comparação da mortalidade por câncer de pulmão em mulheres e homens na cidade de São Paulo de 2005 a 2014.

provou que a idade explica $96 \%$ da mortalidade por câncer de pulmão e brônquios no período estudado $\left(\mathrm{r}^{2}=0,96\right)$.

Quando comparados os sexos obtivemos um resultado com significância estatística $(\mathrm{P}<0,05)$, mostrando que a mortalidade no sexo masculino foi maior que o sexo feminino.

Quanto a faixa etária, no geral ocorreu um aumento do coeficiente de mortalidade ao longo da idade e em especial acima de 50 anos. $\mathrm{O}$ mesmo padrão foi encontrado apenas para homens e mulheres (Figura 2). Além disso, a partir de 50 anos é possível observar que em homens a mortalidade é maior em todas as categorias de idade (Figura 1).

Comparando a mortalidade por distritos administrativos de São Paulo, observamos que as localidades com maiores coeficientes de mortalidade tanto para o sexo feminino, masculino e geral, variam entre: Jardim Paulista, Consolação e Vila Mariana. Enquanto que, os coeficientes mais baixos no geral são os distritos: Anhanguera, Jardim Ângela e Cidade Tiradentes. No sexo feminino os coeficientes menores são: Parelheiros, Grajaú e São Rafael. Já quanto ao sexo masculino são: Anhanguera, Marsilac e Jardim Ângela.

\section{Discussão}

Foi verificado que nos anos de 2005 a 2014 na cidade de São Paulo, houve um aumento na taxa de mortalidade no sexo feminino, fato que pode estar relacionado ao recente aumento da adesão de mulheres ao tabagismo, um hábito cultural e historicamente masculino.

Os mesmos resultados foram constatados por outros autores que evidenciaram uma redução da mortalidade por câncer de pulmão no Brasil para os homens e um aumento para as mulheres ${ }^{(7-8)}$.
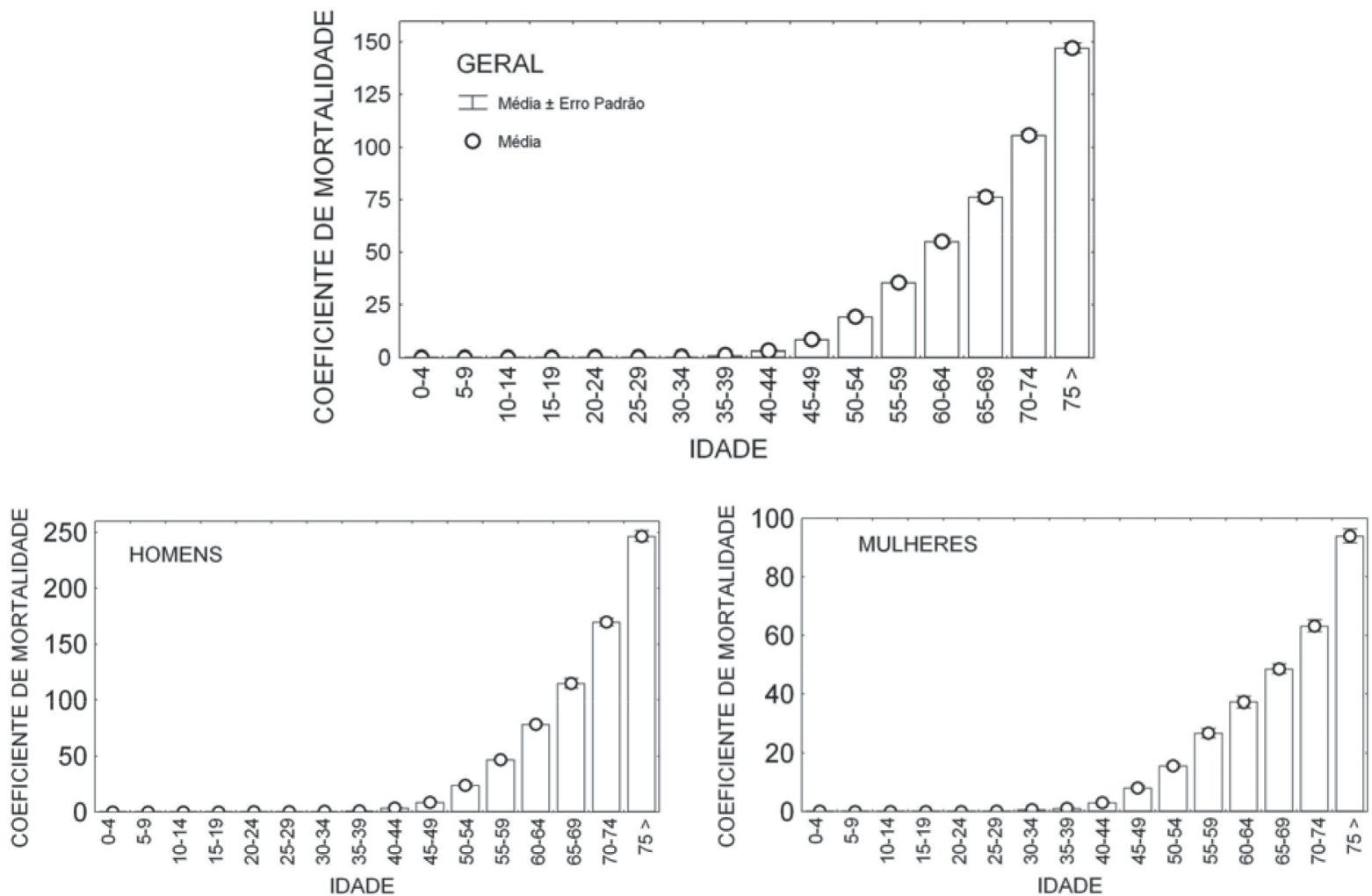

Figura 2 - Comparação da mortalidade por câncer de pulmão e brônquios por faixa etária em pacientes da cidade de São Paulo de 2005 a 2014 
Os distritos de grandes áreas urbanas com maiores rendas per capita, como Jardim Paulista, Consolação e Vila Mariana; foram os que apresentaram um maior número de mortes por essa doença, quando comparados com outros distritos cuja a renda é menor, como Anhanguera, Jardim Ângela e Iguatemi ${ }^{(10)}$.

Este resultado pode estar relacionado à presença de uma maior população nessas áreas e, consequentemente, à uma maior poluição ou até mesmo pelo fato de, sendo regiões onde a renda é maior; terem mais recursos para financiar o hábito de fumar, um dos principais fatores de risco para o desenvolvimento da doença ${ }^{(9)}$.

A mesma constatação foi observada no resultado de outro estudo, onde as regiões mais pobres e mais ricas do planeta foram comparadas. Nesse estudo, conforme aumentava a renda das populações, maior era o número de fumantes ${ }^{(8)}$.

Embora esteja crescendo a quantidade de fumantes em países em desenvolvimento, ainda assim, em países desenvolvidos esse índice é ainda maior ${ }^{(8)}$.

Ainda na análise de dados referentes aos distritos administrativos, naqueles em que há uma renda mais alta, também pode-se observar um maior número de idosos em comparação com os distritos de renda menor.

Tais resultados podem estar associados à antiguidade desses distritos, que tiveram mais tempo de se consolidar como áreas residenciais.

Também foi constatado em outro estudo que ações do governo de conscientização sobre o tabagismo têm prevenido, ou ao menos reduzido, a adesão de jovens ao hábito de fumar, o que pode estar contribuindo para a redução da taxa de mortalidade entre os jovens, se comparado aos idosos, em cujo hábito já está arraigado (7), isso sem mencionar que se trata de doença crônica o que pode explicar o maior número de casos em idosos.

Todas as variáveis estudadas influenciaram a taxa de mortalidade do câncer de pulmão.

Os dados encontraram respaldo na literatura, elencando os principais fatores que determinam a mortalidade da referida patologia, sendo o principal deles, o tabagismo; e sua determinante ação maléfica na etiologia do câncer de pulmão, associado a outros fatores como exposição ocupacional e poluição do ar que pressupõe uma explicação para a diferença dos coeficientes de mortalidade das regiões de São Paulo.

Concluiu-se que fatores individuais como gênero e faixa etária tiveram sua importância na taxa de mortalidade da doença, o primeiro por questões fisiológicas, tabagismo e ocupacional; o segundo, pela vulnerabilidade fisiológica do corpo humano ao longo do tempo. Enquanto aos distritos administrativos, conforme aumenta a renda da população, maior é o número de fumantes, pois são regiões que possuem mais recursos para financiar o hábito de fumar tabaco.

Ficou evidente que, tão logo controlados e/ou evitados os fatores predisponentes do câncer de pulmão, o surgimento da doença torna-se menos incidente.

\section{Referências}

1. Ferlay J, Soerjomataram I, Dikshit R, Eser S, Mathers C, Rebelo $\mathrm{M}$, et al. Cancer incidence and mortality worldwide: sources, methods and major patterns in GLOBOCAN 2012. Int J Cancer. 2015; 136(5):E359-86

2. Brasil. Ministério da Saúde. Instituto Nacional de Câncer José Alencar Gomes da Silva. Coordenação de Prevenção e Vigilância. Estimativa 2014: incidência de câncer no Brasil. Rio de Janeiro; INCA: 2014.

3. Canadian Cancer Society [online] Risk factors for lung cancer. Montreal: Canadian Cancer Society; 2016. Available from: http:/ / www.cancer.ca/en/cancer-information/cancer-type/ lung/risks/?region=on [2016 May 13]

4. Brasil. Ministério da Saúde. Portal da Saúde. Ministério da Saúde regulamenta a Lei Antifumo. [online]. Data de Cadastro: 02/06/2014 as 09:06:52 alterado em 04/07/2014. Disponível em: http://portalsaude.saude.gov.br/index.php/o-ministerio/ principal/secretarias/svs/noticias-svs/13080-ministerio-da-saude-regulamenta-a-lei-antifumo. [10 maio 2016]

5. American Cancer Society. Benefits of quitting smoking over time. [online]. Last Medical Review: 09/09/2016, Last Revised: 09/09/2016. Atlanta (GA): American Cancer Society; 2016. Available from: http://www.cancer.org/healthy/stayawayfromtobacco/benefits-of-quitting-smoking-over-time [Sep 9 2016]

6. World Health Organization. Health statistics and information systems. WHO Mortality Database. [online]. Geneva: WHO; 2016. Available from: http:/ / www.who.int/healthinfo/mortality_data/en/[2016 May 6]

7. Malta DC, Moura L, Souza MFM, Curado MP, Alencar AP, Alencar GP. Tendência de mortalidade do câncer de pulmão, traqueia e brônquios no Brasil, 1980-2003. J Bras Pneumol. 2007; 33(5):536-43.

8. Brasil. Ministério da Saúde. Secretaria de Vigilância em Saúde. Secretaria de Atenção à Saúde. Instituto Nacional de Câncer. Coordenação de Prevenção e Vigilância. Inquérito domiciliar sobre comportamentos de risco e morbidade referida de doenças e agravos não transmissíveis: Brasil, 15 capitais e Distrito Federal, 2002-2003. Rio de Janeiro: INCA; 2004. 184p.

9. Castro MSM, Vieira VA, Assunção RM. Padrões espaço-temporais da mortalidade por câncer de pulmão no Sul do Brasil. Rev Bras Epidemiol. 2004; 7(2):131-43.

10. Fundação Sistema Estadual de Análise de Dados - SEADE. Distribuição dos domicílios, por faixa de Renda per Capita, segundo Distritos do Município de São Paulo. São Paulo: SEADE; 2000. Disponível em: www.produtos.seade.gov.br/produtos/ $\mathrm{msp} / \mathrm{ren} /$ ren2_001.htm [7 maio 2016]

Trabalho recebido: $28 / 06 / 2016$

Trabalho aprovado: 24/10/2017 